\title{
Global sensitivity analysis in a Multi-state Physics Model of Component Degradation based on a hybrid State-Space Enrichment and Polynomial Chaos Expansion approach
}

\author{
Claudio M. Rocco S. ${ }^{*}$, Enrico Zio ${ }^{2,3}$ \\ ${ }^{I}$ Universidad Central de Venezuela, Caracas, Venezuela \\ ${ }^{2}$ Chair on Systems Science and the Energetic Challenge, European Foundation for New Energy- \\ Electricité de France, Ecole Centrale Paris and Supelec \\ ${ }^{3}$ Dipartimento di Energia, Politecnico di Milano, Milano, Italy
}

\begin{abstract}
This paper extends previous works related to the assessment of component degradation, through Markov multi-state physic models. The extension includes the evaluation of the effects of uncertain parameters in the model and the definition of their importance with respect to their influence on the output of the model. Global Sensitivity Analysis (GSA) is selected as the technique because able to: 1) consider the simultaneous effects of parameters variations and 2) to define importance indexes that allow a ranking of the components. GSA requires a large number of evaluations for specific points, identified by an appropriate design of experiment. To avoid the many costly evaluations, a meta-model is built based on polynomial chaos expansion (PCE). A PCE is a multidimensional polynomial approximation of the model with coefficients determined by evaluating the model in a reduced set of predetermined points. Importance indexes values are then derived directly from the PCE. Since in the problem considered, the model provides the timedependent behavior of the state probabilities, the importance indexes are also functions of time. An application is presented, related to the cracking process in an Alloy 82/182 dissimilar metal weld in the primary coolant system of a nuclear power plant.
\end{abstract}

Keywords: Degradation; Global Sensitivity Analysis; Inhomogeneous Continuous Time Markov Chain; Multi-state physics model; Parameter Uncertainty; Polynomial Chaos Expansion; State-space Enrichment.

\section{INTRODUCTION}

Multi-state models (MSM) [1]-[4] have been proposed to assess the degradation processes of components or systems [5]-[6]. In general, a state-space diagram is used

\footnotetext{
1 croccoucv@gmail.com; +58-412-2528346
} 
to define the stochastic process of transition among system states which represent a range of levels from perfect functioning to complete failure.

Then, a model is developed and solved to represent the dynamical behavior of the probability of being in each state [7]-[8]. In general, transition rates are estimated from field data, but "in practice, it can be difficult or even impossible to collect relevant data especially for the highly reliable devices" [9].

Recently, Unwin et al. [10] proposed a multi-state physics modeling approach to assess the degradation processes (e.g. crack growth) of components or systems. The main idea is to describe the transition rates among states by physic functions rather than estimated from service data. As a result transition rates are time-dependent thus defining a non-Markovian model. Several approaches have been devised to solve the non-Markovian problem, such as state-space enrichment approach [10], stochastic Petri net (SPN) [9]-[11] and Monte Carlo simulation [9].

In general, the physic functions used to describe transition rates depend on a set of parameters or factors. For example the micro-crack initiation transition rate $\varphi_{1}$, of the application considered in Section 5, from initial state $S$ to micro-crack state $M$ is defined as: $\varphi_{1}=(b / \tau)(t / \tau)^{b-1}$, where $\tau$ and $b$ are fitting parameters estimated from observed data, and possibly subject to uncertainties.

The existence of uncertainties poses the typical question: What happens to the probability of a specific state if parameters have uncertainty? Moreover, which parameters affect the output of the model the most? In general, these questions are often addressed by analyzing the effect of the variation of a single parameter at a time (also known as Once At a Time evaluation) or using Taylor-series based expansions [12]. However, to account for the possible effects of interactions among parameters (for example between $b$ and $\tau$ in $\varphi_{1}$ ), global sensitivity analysis (GSA) methods are 
required. GSA is known to consider the effect of a factor while all others are varying as well, thus allowing the exploration of the multi-dimensional input space [13] and is able to produce indexes that assess the importance of the factors, taking into account interactions among them.

In general, importance indexes are calculated by evaluating the model output (i.e. the state probabilities for the case of interest here) at a set of specific points derived using random or special sampling techniques from the joint distribution of the space of input factors [14].

GSA methods based on variance decomposition decompose the output variance in contributions of each factor (main effects) and possible interactions. GSA has been used in many applications (e.g., [15]-[16]). Recently, Rocco and Zio [17] applied GSA to assess the importance of transition rates, in homogeneous continuous Markov models of power system components.

The number of model evaluations required for a GSA depends on the number of factors in the model and the sample size. The evaluation of the indexes depends on the complexity of the model and could require very large computational times. In these cases, it is possible to use surrogate or meta-models that approximate the original model performance. Several techniques have been suggested to build such metamodels, like regression models, artificial neural networks or support vector machines, among others.

In this paper, a particular technique named Polynomial Chaos Expansion (PCE) is selected to create a meta-model represented by a polynomial of the factors. If the factor uncertainties are modeled as random variables with known probability density functions, then the coefficients of the resulting polynomial contain "the complete 
response of the model" [18]. In this way, factor importance indexes can be assessed directly from the coefficients of the polynomial decomposition.

PCE has been used in many areas including GSA [19]-[34]. To our best knowledge it has not been used to assess importance indexes in multi-state physics models of component degradation.

In this paper, we extend the use of GSA in Markov models [17] to multi-state physics models whose solutions are not known analytically and thus obtained by numerical methods.

Note that multi-state physics models assess the behavior of the system under analysis as a function of time. For this reason, in this paper, the PCE of a dynamic system, recently suggested in [18] is selected. As a result, a PCE is used at each time in order to derive the dynamical behavior of the importance indexes of the factors.

The presentation of the work is structured in the paper as follows: Section II defines the Markov model setting. Section III briefly reviews some SA concepts, while Section IV provides a brief introduction to PCE. Section V presents results of experimentation and Section 6 presents conclusions and future work.

Acronyms, Notations and Assumptions

$\begin{array}{ll}\text { Acronyms } & \\ \text { CTMC } & \text { : Continuous Time Markov Chain } \\ \text { DOE } & \text { : Design Of Experiment } \\ \text { gPC } & \text { : generalized Polynomial Chaos } \\ \text { GSA } & \text { : Global Sensitivity Analysis } \\ \text { IM } & \text { : Importance Measure } \\ \text { LHS } & \text { : Latin Hypercube Sampling } \\ \text { OAT } & \text { : One factor at A Time } \\ \text { MSM } & \text { : Multi-state models } \\ \text { PC } & \text { : Polynomial Chaos } \\ \text { PCE } & \text { : Polynomial Chaos Expansion } \\ \text { pdf } & \text { : probability density function } \\ \text { SA } & \text { : Sensitivity Analysis }\end{array}$




\section{Notation}

$\xi \quad$ vector of random variables

$\xi_{\mathrm{i}} \quad$ i-th random variable with a pdf defined

$\Phi_{i}() \quad$ polynomial of the selected basis.

$a_{\mathrm{i}} \quad$ i-th PCE coefficient

$d$ polynomial degree

$m$ number of states

$n i \quad$ number of integration points

$M+1$ Number of coefficients in the PCE

$n \quad$ number of random factors

$o q$ order of the Smolyak algorithm

$\mathrm{P}(t) \quad$ Transient probability vector $\left(\mathrm{P}_{1}(t), \mathrm{P}_{2}(t), \ldots \mathrm{P}_{m}(t)\right)^{\mathrm{t}}$

$\mathrm{P}_{i}(t) \quad$ Transient probability of state $i$

$Q(t) \quad$ infinitesimal generator matrix

$\mathrm{q}_{i j}(t)$ transition rate from state $\mathrm{i}$ to state $\mathrm{j}$

$S_{i} \quad$ main order sensitivity IM for factor $i$

$S_{T i} \quad$ total order sensitivity IM for factor $i$

\section{Assumptions}

1. The number of states in the Markov models is finite

\section{MARKOV MODELING}

The evolution in time of a system which changes state stochastically as its components change modes of operation at random times (due to degradation, reduced production, failure, repair, etc.) can be mathematically described by a discrete-state, continuous-time Markov chain (CTMC) $Z=\{z(t), t \geq 0\}$, with finite state space $\mathrm{E}=\{1,2, \ldots, m\}$.

Let $Q(t)$ be the $m \times m$ infinitesimal generator matrix whose generic element $\mathrm{q}_{i j}(t)$ represents the transition rate from state $i$ to state $j(i, j \in \mathrm{E}), \mathrm{q}_{i i}(\mathrm{t})=\sum_{\mathrm{i} \neq \mathrm{j}} \mathrm{q}_{i j}(\mathrm{t})$, and $P_{i}(t)$ be the probability that the system is in state $i$ at time $t$. The $m$-dimensional probability vector $P(t)$ is obtained by solving:

$$
\dot{P}(t)=P(t) Q(t)
$$

given initial conditions for $P(0)$. 
In general, transition rates can be constant (homogenous Markov Chain) or functions of time (non-homogeneous Markov Chain). General techniques for solving Ordinary Differential Equations, like Euler or Runge-Kutta methods [35], the special implicit method [36] in case of stiff models or uniformization-based algorithms can be used for solving (1) [37].

In some models, as the one of Section 5, some transition rates are functions of the holding times in each state. In these cases, the previous general methods can not be used directly. As suggested in [9]-[10] one of the possible techniques that can be used is the method of state enrichment. Basically the method is based on the "discretization of the component lifetime into equally sized time intervals, during each of which the transition rate remains constant". The new enriched state-space is described by the original states and the vector of discretized times at each state. For example, given a component with 6 original states and a lifetime of 100 years discretized in time steps of 0.5 years, the approximation method would generate a transition matrix with $(6 x(100 / 0.5))^{2}$ entries.

In any case, no matter the solution technique used for solving (1), the numerical values of $\mathrm{P}_{\mathrm{i}}(t)$ are non-linear functions of the transition rates $\mathrm{q}_{i j}(t)$ with possible interactions among them. To evaluate the influence of transition rate uncertainties on $\mathrm{P}_{\mathrm{i}}(t)$ would mean to consider the variability in their values and the corresponding variability in the model state probabilities output. It is also of interest to identify, through a sensitivity analysis, those transition rates most influential in terms of the variations that they induce on the state probabilities when their variability is considered. 


\section{SENSITIVITY ANALYSIS}

Sensitivity analysis (SA) is "the study of how uncertainty in the output of a model (numerical or otherwise) can be apportioned to different sources of uncertainty in the model input factors" $\mathrm{X}=\left(x_{1}, x_{2}, \ldots, x_{n}\right), n$ being the number of factors in the model [14].

Global sensitivity analysis (GSA) considers the exploration of the multidimensional input space [13] and is able to determine the effect of a factor while all others are varying as well. Some of the methods developed, such as Fourier Amplitude Sensitivity Technique (FAST), extended FAST [14] provide a numerical assessment of the importance of each the factor and are able to consider and quantify the interaction among them.

In this paper, variance-based sensitivity indexes are evaluated. The main idea is to decompose the variance of an output as a function of the main effects of each factor and possible interactions.

Let $Y=\mathrm{f}\left(x_{1}, x_{2}, \ldots, x_{\mathrm{n}}\right)$, be a scalar output and $x_{i}$ the $n$ orthogonal input factors treated as random variables (i.e. uncertainty is characterized in terms of a probability density function (pdf)). The variance $V(Y)$ of the output $Y$ can be written as [14]:

$$
V(Y)=\sum_{i} V_{i}+\sum_{i} \sum_{j>i} V_{i j}+\sum_{i} \sum_{j>i} \sum_{l>j} V_{i j l}+\ldots . V_{12 \ldots n}
$$

where:

$V_{i}=V\left(E\left(Y \mid X_{i}\right)\right)$ is the main effect (or first-order term) due to $x_{\mathrm{i}}$, $V_{i j}=V\left(E\left(Y \mid X_{i}, X_{j}\right)\right)-V_{i}-V_{j}$ is the two-way interaction between $x_{\mathrm{i}}$ and $x_{\mathrm{j}}$, and so on.

Main and total order sensitivity indexes can then be defined as [14],[38]:

$$
S_{i} \equiv \frac{V_{i}\left(E_{-i}\left(Y \mid X_{i}\right)\right)}{V_{Y}}
$$


$S_{T i} \equiv \frac{V_{Y}-V_{i}\left(E_{-i}\left(Y \mid X_{i}\right)\right)}{V_{Y}}=\frac{E_{-i}\left(V_{i}\left(Y \mid \mathbf{X}_{-i}\right)\right)}{V_{Y}}$

where $\mathrm{X}_{-i}=\left\{x_{1}, x_{2}, \ldots x_{\mathrm{i}-1}, x_{\mathrm{i}+1}, \ldots x_{\mathrm{n}}\right)$ and $\mathrm{E}_{-i}\left(Y \mid x_{\mathrm{i}}\right)$ is the expected value of $Y$ conditioned on $x_{\mathrm{i}}$, thus being a function of factor $x_{\mathrm{i}}$ alone. The final expression in (4) is derived from the fact that, given two random variables $A$ and $B$ : $V(A)=E(A \mid B)+V(E(A \mid B))[14]$.

The main index $S_{i}$ is the fraction of variance of the output $V(Y)$ that can be attributed to $x_{\mathrm{i}}$ alone, while $S_{T i}$ corresponds to the fraction of $V(Y)$ that can be attributed to $x_{i}$ including all its interactions with other factors [14]. For example, in the case of 3 factors $x_{1}, x_{2}$ and $x_{3}: S_{T 1}=S_{1}+S_{12}+S_{13}$.

That means that "the computation of total sensitivity indices is a shortcut to measure the overall effect of "main effect" and all higher order interactions" [13].

The main index $S_{i}$ is the measure to be used to determine which subset of parameters accounts for most of the output uncertainty [38], while $S_{T i}$ must be used as a screening tool for identifying the subset of non-influential factors, i.e. factors that when fixed to any value within their uncertainty range, do not significantly reduce the output variance [38]. The difference $\left(S_{T i}-S_{i}\right)$ assesses the interaction effects of a factor.

The estimation of $S_{i}$ and $S_{T i}$ are approximated by assuming independence among factors and using random or special sampling techniques (e.g., Monte Carlo simulation based on Latin Hypercube Sampling (LHS) approach from the joint distribution of the space of input factors). Techniques such as Sobol or extended FAST could be used to determine $S_{i}$ and $S_{T i}$ [14]. One of the main disadvantages of this approach is that it requires several model evaluations and, thus, can be computationally time consuming. For example, the "extended-FAST" method [39] used to estimate first-order and total indices for all the $n$ factors of a model, has a total 
computational cost of ( $n s \times n)$ model evaluations, where $n s$ is an integer (that can vary "from a few hundreds to a few thousands" [40]) representing the sample size selected and depends on the convergence properties of the sensitivity indexes. Thus, depending on the number of factors and the computational complexity of the model, the technique could require very large computational times.

\section{POLYNOMIAL CHAOS EXPANSION}

\section{A. Introduction}

The basic idea of PCE is to approximate the outputs of a system (e.g., state probabilities in our case) through an orthonormal polynomial basis in the uncertain parameter spaces (e.g., transition rates). Wiener [41] was the first to propose the use of Polynomial Chaos (PC), when parameters follow a Gaussian distribution. In this case, the basis is defined through Hermite polynomials. However, this limitation was extended to generalized polynomial chaos (gPC), which is able to consider more distributions [42]. Recently [30] defines the arbitrary polynomial chaos (aPC), which generalizes PC and is able to accommodate arbitrary distributions or distributions known with a few statistical moments.

Let $\xi=\left[\xi_{1}, \ldots, \xi_{n}\right]$ define the vector of $n$ random input factors and $\Omega(\xi)=f(\xi)$ a secondorder with finite variance model output. PC theory [42] shows that the spectral expansion the model output $\Omega(\xi)$ can be expanded as:

$\Omega(\xi)=\sum_{i=0}^{\infty} a_{i} \Phi_{i}\left(\xi_{1}, \ldots, \xi_{n}\right)$

where:

$a_{i}$ are the coefficients of the expansion

$\xi_{\mathrm{i}}$ are random variables with a pdf defined

$\Phi_{i}()$ are polynomials of the selected basis. 
The polynomial basis is selected based on the pdf of $\xi_{i}$. As an example, if $\xi_{\mathrm{i}}$ are standard normal random variables, then the basis is defined as multivariate Hermite polynomials. Table 1 shows the type of distribution and the associated polynomials as suggested in the Wiener-Askey scheme [42].

Table 1: Relation between probability distribution and polynomial basis

\section{based on the Wiener-Askey scheme [42]}

For example, if $\varepsilon$ is a standard normal random variable, the Hermite polynomials up to third order are:

\begin{tabular}{cc}
$k$ & $\mathrm{H}_{k}(\varepsilon)$ \\
\hline 0 & 1 \\
\hline 1 & $\varepsilon$ \\
\hline 2 & $-1+\varepsilon^{2}$ \\
\hline 3 & $-3 \varepsilon^{3}+\varepsilon^{3}$ \\
\hline
\end{tabular}

For practical purpose, the infinite series in (2) is limited to a finite number of terms $M+1$ which depends on $n$, the number of random variables of the model and on the selected polynomial degree $d: M+1=\frac{(n+d) !}{n ! d !}[18]$. For example, if $n=2$ and $d=3$, then $M+1=10$.

The multivariate polynomial $\Phi_{i}()$ is given by the tensor product of the corresponding one dimensional selected polynomial [18]. For example, if $\varepsilon_{1}$ and $\varepsilon_{2}$ are standard normal random variables, the 10 terms of the PCE expansion for $\Omega\left(\xi_{1}, \xi_{2}\right)$ up to third order is given by [43]:

$$
\begin{aligned}
& \Omega\left(\xi_{1}, \xi_{2}\right)=a_{0}+a_{1} \xi_{1}+a_{2} \xi_{2}+a_{3}\left(\xi_{1}^{2}-1\right)+a_{4}\left(\xi_{2}^{2}-1\right)+a_{5} \xi_{1} \xi_{2}+a_{6}\left(\xi_{1}^{3}-3 \xi_{1}\right)+ \\
& a_{7}\left(\xi_{2}^{3}-3 \xi_{2}\right)+a_{8}\left(\xi_{1} \xi_{2}^{2}-\xi_{1}\right)+a_{9}\left(\xi_{2} \xi_{1}^{2}-\xi_{2}\right)
\end{aligned}
$$


The terms $a_{\mathrm{i}}$ are unknown and must be determined. There are two approaches for their evaluation: the first is based on the modification of the model $\Omega(\xi)$ and its solution (intrusive approach) while the second does not require any modification of the model equations (non-intrusive approach). In the latter approach, the terms are estimated from the evaluation of the model under study in a set of selected samples of the random vector $\xi$. In our case, the set of differential equations (1) is solved, based on a set of transition rate deviates.

Two techniques are described in the literature for the non-intrusive approach. The first (regression method) is based on the optimization of the mean-square difference between the model and the approximation [24]. The optimization is performed on a set of $\mathrm{N}=2(M+1)$ samples $\xi_{j}$ and its corresponding evaluation $\Omega\left(\xi_{j}\right)$. The second technique is based on projection methods which exploit the orthogonality properties of the PC basis. In fact, by evaluating the inner product of the PC expansion (equation (2)) with $\Phi_{l}(\xi)$ :

$\left\langle\Omega(\xi), \Phi_{l}(\xi)\right\rangle \approx \sum_{i=0}^{M} a_{i}\left\langle\Phi_{i}\left(\xi_{1}, \ldots, \xi_{n}\right), \Phi_{l}\left(\xi_{1}, \ldots \xi_{n}\right)\right\rangle$

where $\langle\cdot, \cdot\rangle$ is the inner product in the Hilbert space, and taking into account the orthogonality of the basis, the coefficients can be obtained as [18]:

$a_{i}=\frac{\left\langle\Omega\left(\xi_{1}, \ldots, \xi_{n}\right), \Phi_{i}\left(\xi_{\left.1, \ldots, \xi_{n}\right)}\right)\right.}{\left\langle\Phi_{i}\left(\xi_{1}, \ldots, \xi_{n}\right), \Phi_{i}\left(\xi_{\left.1, \ldots, \xi_{n}\right)}\right)\right.}$

The numerator in (4) is computed as:

$$
\left\langle\Omega\left(\xi_{1}, \ldots, \xi_{n}\right), \Phi_{i}\left(\xi_{1}, \ldots, \xi_{n}\right)\right\rangle=\int_{d} \Omega\left(\xi_{1}, \ldots \xi_{n}\right) \Phi_{i}\left(\xi_{1, \ldots, \xi_{n}}\right) h\left(\xi_{1, \ldots, \xi_{n}}\right) d\left(\xi_{1, \ldots, \xi_{n}}\right)
$$

where $h\left(\xi_{1}, \ldots, \xi_{n}\right)$ is the joint probability density function of $\xi$. 
As mentioned in [18], the coefficients could be obtained by solving up to $(M+1)$ multidimensional integrals (5). However, the integrals are numerically approximated by evaluating $\Omega\left(\xi_{\mathrm{j}}\right)$ in a set of selected $n i$ integration points $\xi_{j}(j=1, . ., n i)$, using the following general expression [18]:

$\int_{d} \Omega\left(\xi_{1}, \ldots, \xi_{n}\right) \Phi_{i}\left(\xi_{1}, \ldots, \xi_{n}\right) h\left(\xi_{1}, \ldots, \xi_{n}\right) d\left(\xi_{1}, \ldots, \xi_{n}\right)=\sum_{j=1}^{n i} \Omega\left(\xi_{1}^{(j)}, \ldots \xi_{n}^{(j)}\right) \Phi_{i}\left(\xi_{1}^{(j)}, \ldots \xi_{n}^{(j)}\right) w^{(j)}$

where $w^{(\mathrm{j})}$ are specific weights. This means that the computational cost for determining the polynomial coefficients are proportional to the number of integration points $n i$.

There are two approaches to solve the multidimensional integrals: Deterministic and Probabilistic algorithms [44]. The first ones are based on high order interpolation schemes. For example, the Smolyak algorithm [45] of order oq uses an interpolation approach by evaluating polynomials from lower dimensions to higher order at selected points. Figure 1 shows the set of points generated by the algorithm for $d=2$ and $o q=4$ (33 points) and $o q=6$ (65 points). Note that the set of points for $o q=4$ is included in the set of points for $o q=6$.

The second, like Monte Carlo methods, uses random samples. In both approaches, the convergence of the integrals depends on the smoothness of the model under analysis $[25]$.

As suggested in [25], the Smolyak approach is, in general, a convenient method when the number of factors is less than 15 , while Monte Carlo methods are "best suited for higher dimensional problems". In this paper, the Petras-technique [45], a special Smolyak-based method is used, as implemented in the Scilab toolbox NISP [46].

After determining the coefficients, the polynomial meta-model is already defined. As shown in [24] and [25] sensitivity indexes for all factors, including $S_{i}$ and $S_{T i}$ are 
evaluated analytically from the polynomial coefficients. So the computational cost for deriving them corresponds basically to the cost required to evaluate numerically a set of multidimensional integrals.

\section{B. PCE for dynamical systems}

The PCE of dynamical systems has been recently proposed in [18]. Since the output of the model is a function of time, the PCE is based on coefficients that also vary as a function of time. This means that at each time instant a set of parameters are required. If $\Omega=f(\xi, t)$ then $[18]$ :

$\Omega(\xi, t) \approx \sum_{i=0}^{M} a_{i}(t) \Phi_{i}\left(\xi_{1}, \ldots, \xi_{\mathrm{n}}\right)$

As in the previous subsection, each time the variance decomposition is performed analytically from the polynomial coefficients, all sensitivity indexes are determined for each parameter in the model.

Based on the two-step procedure proposed in [18], the following approach is defined:

1. Based on the parameter distribution, select the corresponding polynomial type

2. Define the polynomial degree $d$

3. Calculate $M+1$

4. For $t=0$ until a specific time limit $\mathrm{t}_{\max }$ \{

5. Estimate the PC coefficients

6. Calculate sensitivity indexes for each parameter \}

In step 2, the selection of the appropriate degree $d$ could be done iteratively, that is, trying different values for $d$ and evaluating the average relative error between the model output and the PCE approximation [18]. 


\section{EXAMPLE: A NUCLEAR COMPONENT UNDERGOING STRESS CORROSION CRACKING}

This example refers to the cracking process in an Alloy 82/182 dissimilar metal weld in a primary coolant system of a nuclear power plant, previously analyzed by [9]-[10] using a multi-state physics model. Figure 2 shows the six-state transition diagram model.

Figure 2: Transition diagram of the multi-state physics model of crack development in Alloy 82/182 dissimilar metal welds [9]

Transition rates among states are defined as: $\varphi_{1}$ is the micro-crack initiation transition rate, $\varphi_{2}, \varphi_{3}$ and $\varphi_{4}$ are crack growth transition rates, $\varphi_{5}$ and $\varphi_{6}$ are transition rates that lead to ruptures, $\omega_{1}, \omega_{2}, \omega_{3}$ and $\omega_{4}$ are transition rates associated with detection/repair at the different states. In [10], the transition rates $\varphi_{1}, \varphi_{2} \varphi_{3}$ and $\varphi_{4}$ are time-dependent and/or stochastic, whereas the others are assumed constant.

An interesting fact in this example is that some transition rates depend on the time the system is in a particular state. For example, the transition rate $\varphi_{2}$ (between state $M$ and $D$ ) describes the transitions from micro-crack state $M$ to radial-crack state $D$ and is defined as a function of $u$, the time of crack initiation.

The expressions for transition rates $\varphi_{1}, \varphi_{2} \varphi_{3}$ and $\varphi_{4}$ are presented in Table 2. As shown in Table 2, the transition rates are function of a set of 14 factors (i.e., $n=14$ ) whose base values are shown in Table 3 .

Table 2: Expressions for transitions rates $\varphi_{1}, \varphi_{2} \varphi_{3}$ and $\varphi_{4}$

Table 3: Parameter definition and base value

Figure 3 shows the time-dependent behavior of $\mathrm{P}_{\mathrm{i}}(t)$ for each state, computed using the state enrichment method for a component lifetime $t_{\max }=80$ years and a time step of 1 year [9]. 
Figure 2: Transient behavior for $P_{i}(t)$, base case [9]

As observed in [9], "1) there is an early, rapid transition from the Initial state to the Micro-crack state; 2) there is a monotonic increase in the probability of the Rupture state". In the time span 5-20 years it is possible to detect a transient behavior.

For the importance indexes assessment, a $\pm 10 \%$ variation on base values is assumed described by uniform distributions. Importance sensitivity indexes are derived using the PCE approach, based on a Legendre polynomial basis as suggested from Table 1. Note that each factor must be mapped on the interval $[-1,1]$.

In this paper, a non-intrusive approach is selected: State probabilities $\mathrm{P}_{i}(t)$ are evaluated using the state enrichment method as the "black-box" model. The PCE as well as the sensitivity indexes are evaluated using the Scilab toolbox NISP [46] and a Smolyak integration technique is selected based on Petras integration points [45].

As suggested in [18], different values for $d$ were selected and the average relative error between the model output and the PCE approximation were evaluated. For $d=3$ the PCE is considered sufficient since the maximum error is less than $6.2 \%$, as shown in Figure 4. In this case, the number of models evaluations is equal to 3361 [47] and the PCE expansion has 681 terms.

Figures 5-7 show the time-dependent behavior of $S_{i}, S_{T i}$ along with the value of $\left(S_{T i}\right.$ $S_{i}$ ) for each factor considered and for each state probability. The title in each figure is coded as: sx PETRAS yy z-ww,

where:

$\mathrm{x}$ is the state considered yy is the type of index presented $\mathrm{z}$ is the polynomial degree ww is the number of model evaluations (PETRAS stands for the option selected in NISP [46] for the determination of the $n i$ integration points) 
Let us consider the time-dependent behavior of the sensitivity indexes related to the initial state $\mathrm{S}$ (state 1). As mentioned in Section $\mathrm{C}, S_{i}$ is used to determine which parameters account for most of the output uncertainty, $S_{T i}$ is used for identifying the non-influential factors, and $\left(S_{T i}-S_{i}\right)$ for quantifying interaction effects. To perform the analysis the following criterion was used: if $S_{i}$ or $S_{T i}$ or $\left(S_{T i}-S_{i}\right)<0.1$ then the corresponding index is not considered.

The analysis reveals:

1) Top left panel in Figures 5: During the component lifetime, parameter $\tau$ accounts for most of the output uncertainty. However in the time span 8-10 parameter $\mathrm{a}_{\mathrm{C}}$ accounts for most of the output. For $\mathrm{t}>20, \tau$ and $\omega_{1}$ are the most important parameters.

2) Top left panel in Figures 6: Several parameters have low $S_{T i}$ values, indicating that they are non-influential factors

3) Top left panel in Figure 7: The index $\left(S_{T i}-S_{i}\right)$ reflects the interaction effects between a factor and all the others. In general the index is small (i.e., $S_{T i} \approx S_{i}$ ) during the component lifetime. However in the time span 5-10, there are interactions between $\tau$ and the rest of the parameters.

A similar analysis can performed for the other states. In general, the importance of each factor is dependent not only on the state considered but also at what time is evaluated.

The index $S_{i} \geq 0.1$ is used to determine which parameters account for most of the output uncertainty. Table 3 shows the important factors for each probability state during the time-span simulated.

\section{CONCLUSIONS}

The output of a model depends on the input parameters. In many situations, inputs are not precisely known because subject to uncertainty. It is important to know how uncertainty in the parameters affects the results of the model. Such assessment is possible using global sensitivity analysis, an approach able to analyze the variance of the output and determine the individual effects of each parameter (factor) as well as 
the possible interactions among them. In practice, global sensitivity analysis requires many evaluations of the model which may pose a problem of computation time. In order to reduce the computation burden, a surrogate model can be constructed.

This paper presents a hybrid approach for evaluating the importance of uncertainty factors in multi-state physics models of component degradation.

The original contributions of the paper are : a) the assessment of the effects of uncertain parameters in a Markov multi-state physics model of component degradation and of their importance with respect to the model output by using GSA; and b) the use of Polynomial Chaos Expansion to derive an efficient meta-model for coping with the computational cost of the analysis.

An example is presented related to a cracking process described by a six-state Markov model with 14 uncertain factors, The meta-model is based on a PCE of third order whose coefficients are determined using only 3341 model evaluations. The metamodel is able to mimic the dynamic behavior of the degradation state probabilities, with a maximum average relative error less than $6.2 \%$. The sensitivity indexes for each state reveal that the ranking of importance is time-varying and each state has its own set of important factors.

Future works aim to test some approaches for: 1) reducing the number of evaluations without affecting the accuracy of the expansion, for example, through an initial screening approach, as the one suggested in [24];2) evaluating the effects of different probability distributions associated to the uncertain factors; 3) considering the robustness of the importance indexes when modeling dependencies among factors; and 4) The convenience of using an integrated importance index able to consider simultaneously the effects of one factor in several outputs. 


\section{REFERENCES}

1. Kuo, W. and Zuo, M. J., Optimal Reliability Modeling: Principles and Applications. New York: John Wiley \& Sons, 2003.

2. Lisnianski, A. and Levitin, G., Multi-state System Reliability: Assessment, Optimization and Applications. Singapore: World Scientific, 2003.

3. Li, W. J. and Pham, H., "Reliability modeling of multi-state degraded systems with multi-competing failures and random shocks," IEEE Transactions on Reliability, vol. 54, pp. 297-303, 2005.

4. Levitin, Gregory Optimal Structure of Multi-State Systems With Uncovered Failures, , IEEE Transactions on Reliability, vol. 57, pp. 140-148, 2008.

5. Park, C. and Padgett, W. J., "Stochastic degradation models with several accelerating variables " IEEE Transactions on Reliability, vol. 55, pp. 379-390, 2006.

6. Gebraeel, N., Elwany, A., and Pan, J., "Residual Life Predictions in the Absence of Prior Degradation Knowledge," IEEE Transactions on Reliability, vol. 58, pp. 106-117, 2009.

7. Billinton R., Allan R.: Reliability Evaluation of Engineering Systems, Second Edition, Plenum Press 1992.

8. Zio E.: Computational Methods For Reliability And Risk Analysis, Series on Quality, Reliability \& Engineering Statistics Vol 14, World Scientific Publishing Company, 2009.

9. Li Y., Zio E., A Multi-state Physics Model of Component Degradation based on Stochastic Petri Nets and Simulation, IEEE Transactions on Reliability, vol. 61, pp. 921-931, 2012.

10. Unwin, S. D., Lowry, P. P., Layton, R. F., Heasler, P. G., Toloczko, M. B.: Multistate physics models of aging passive components in probabilistic risk assessment, in Proceedings of ANS PSA 2011 International Topical Meeting on Probabilistic Safety Assessment and Analysis, pp. 1-12, 2011.

11. Schneeweiss, W. G., "Tutorial: Petri nets as a graphical description medium for many reliability scenarios," IEEE Transactions on Reliability, vol. 51, pp. 39-48, 2002.

12. Havekort, B. and Meewissen, A. Sensitivity and uncertainty analysis of Markovreward models. IEEE Transactions on Reliability, vol. 44, pp. 147-153, 1995.

13. Campolongo F., Saltelli A., Cariboni J.: From screening to quantitative sensitivity analysis. A unified approach, Computer Physics Communications 182, 978-988, 2011.

14. Saltelli A., Chan K., Scott E.M., Sensitivity Analysis, John Wiley \& Sons, Probability and Statistics series, 355-365, 2000.

15. Tarantola S., Kopustinskas V., Bolado-Lavin R, Kaliatka,A., Ušpuras,E., Vaišnoras, M., Sensitivity analysis using contribution to sample variance plot: Application to a water hammer model, Reliability Engineering and System Safety, Volume 99, Pages 62-73, 2012.

16. Xu M., Chen T., Yang,X., The effect of parameter uncertainty on achieved safety, integrity of safety system, Reliability Engineering and System Safety, Volume 99, Pages 15-23, 2012.

17. Rocco C.M., Zio E.: Global sensitivity analysis in Markov reliability models of power system components, submitted to Second International Conference on Vulnerability and Risk Analysis and Management (ICVRAM2014) 
18. Haro E., Anstett-Collin F., Basset M., Sensitivity study of dynamic systems using polynomial Chaos, Reliability Engineering and System Safety, 104, pp. 15-26, 2012.

19. Ghanem R., Spanos P. D., Polynomial chaos in stochastic finite elements, Journal of Applied Mechanics, 57, 197-202, 1990

20. Xiu D., Karniadakis G. E., Modeling uncertainty in flow simulations via generalized polynomial chaos, Journal of Computational Physics, 187, 137-167. 2003.

21. Lucor D., Enaux C., Jourdren H., Sagaut P., Stochastic design optimization: Application to reacting flows. Computer Methods in Applied Mechanics and Engineering, Volume 196, Issues 49-52, Pages 5047-5062, 2007.

22. Agarwal N., Aluru N.R., Stochastic modeling of coupled electromechanical interaction for uncertainty quantification in electrostatically actuated MEMS, Computer Methods in Applied Mechanics and Engineering, Volume 197, Issues 43-44, Pages 3456-3471, 2008.

23. Ghanem R.G., Doostan A., Red-Horse J., A probabilistic construction of model validation, Computer Methods in Applied Mechanics and Engineering, Volume 197, Issues 29-32, Pages 2585-2595, 2008.

24. Sudret B., Global sensitivity analysis using polynomial chaos expansions, Reliability Engineering and System Safety, 93, 7, 964-979., 2008.

25. Crestaux T., Maitre O. L, Martinez J., Polynomial chaos expansion for sensitivity analysis, Reliability Engineering and System Safety, 94, 1161-1172, 2009.

26. Blatman G., Sudret B., Efficient computation of global sensitivity indices using sparse polynomial chaos expansions, Reliability Engineering and System Safety 95, 11, $1216-1229,2010$.

27. Soize C., Identification of high-dimension polynomial chaos expansions with random coefficients for non-Gaussian tensor-valued random fields using partial and limited experimental data, Computer Methods in Applied Mechanics and Engineering, Volume 199, Issues 33-36, Pages 2150-2164, 2010.

28. Simon F., Guillen P., Sagaut P., Lucor D., A gPC-based approach to uncertain transonic aerodynamics, Computer Methods in Applied Mechanics and Engineering, Volume 199, Issues 17-20, Pages 1091-1099, 2010.

29. Poëtte G., Després B., Lucor D., Treatment of uncertain material interfaces in compressible flows, Computer Methods in Applied Mechanics and Engineering, Volume 200, Issues 1-4, Pages 284-308, 2011.

30. Oladyshkin S., Class H., Helmig R., Nowak W., A concept for data-driven uncertainty quantification and its application to carbon dioxide storage in geological formations, Advances in Water Resources 34, 1508-1518, 2011.

31. Buzzard G. T., Global sensitivity analysis using sparse grid interpolation and polynomial chaos, Reliability Engineering and System Safety 107, 82-89, 2012.

32. Rahman S., Global sensitivity analysis by polynomial dimensional decomposition, Reliability Engineering and System Safety 96, 7, 825- 837, 2011.

33. Constantine P.G., Eldred M.S., Phipps E.T., Sparse pseudo-spectral approximation method, Computer Methods in Applied Mechanics and Engineering, Volumes 229-232, Pages 1-12, 2012.

34. Tootkaboni M., Asadpoure A., Guest J.K., Topology optimization of continuum structures under uncertainty - A Polynomial Chaos approach, Computer Methods in Applied Mechanics and Engineering, Volumes 201-204, Pages 263-275, 2012. 
35. Tombuyses, B., Devooght, J.: Solving Markovian systems of O.D.E. for availability and reliability calculations. Reliability Engineering and System Safety, 48, 47-55, 1995.

36. Hosea M. E., Shampine L. F., Analysis and implementation of TR-BDF2, Appl. Numer. Math. 20 (1-2), 21-37, 1996.

37. van Moorsely A.P.A., Wolterz K.: Numerical Solution of Non-Homogeneous Markov Processes through Uniformization, Proceedings of the 12th European Simulation Multiconference on Simulation - Past, Present and Future, SCS Europe, Pages 710 - 717, 1998.

38. Saltelli, A., Tarantola, S., Campolongo, F., Ratto, M.: Sensitivity Analysis in Practice. A Guide to Assessing Scientific Models, John Wiley \& Sons, Probability and Statistics series, 2004.

39. Saltelli, A., Tarantola, S., Chan, K.: A quantitative, model independent method for global sensitivity analysis of model output, Technometrics, 41, 39-56, 1999.

40. Saltelli, A., Ratto, M., Andres, T., Campolongo, F., Cariboni, J., Gatelli, D. Saisana, M., and Tarantola, S.: Global Sensitivity Analysis: The Primer John Wiley \& Sons, 2008.

41. Wiener N., The homogeneous chaos, American Journal of Mathematics 60, 4, 897-936, 1938.

42. Xiu D., Karniadakis G., The Wiener-Askey polynomials chaos for stochastic Differential equations, Journal of Scientific Computing 26 Volume 24, 619 - 644, 2002.

43. Liang B., Huang S. P., Phoon K. K.: An EXCEL add-in implementation for collocation-based stochastic response surface method, ISGSR2007 First International Symposium on Geotechnical Safety \& Risk, Oct. 18 19, Shanghai, Tongji University, China, 2007.

44. Panayirci H.M, Schuëller.G.I., On the Capabilities of the Polynomial Chaos Expansion Method within SFE Analysis-An Overview, Arch Comput Methods Eng 18: 43-55, 2011.

45. Petras K., Smolyak cubature of given polynomial degree with few nodes for increasing dimension, Numerische Mathematik, Volume 93, Number 4, Pages 729-753, 2002

46. Baudin M., Martinez J., Polynômes de chaos sous Scilab via librairie NISP, in: 42 emes Journees de Statistique, 2010.

47. Heiss F., Winschel V., Likelihood approximation by numerical integration on sparse grids, Journal of Econometrics, Volume 144, Pages 62-80, 2008.

\section{Biographies}

Claudio M. Rocco received the Electrical Engineering and MSc. Electrical Engineering (Power System) degrees from Universidad Central de Venezuela (1980, 1982) and Ph.D. degree from The Robert Gordon University, Aberdeen, Scotland, UK (2000). He is a Full Professor at Universidad Central de Venezuela, currently at Operation Research post-graduate courses. His main areas of research interest are Statistics, Reliability, Evolutionary Multi-objective Optimization and Machine Learning techniques. He has published more than 170 refereed manuscripts related to these areas in technical journals, book chapters, conference proceedings and industry reports. 
Enrico Zio (M'06-SM'09) (B.S. in nuclear engineering, Politecnico diMilano,1991; M.Sc. in mechanical engng., UCLA, 1995; Ph.D., in nuclear engng., Politecnico di Milano, 1995; Ph.D., in nuclear engng., MIT, 1998) is the Director of the Graduate School of the Politecnico di Milano, full Professor of Computational Methods for Safety and Risk Analysis. He is the Chairman of the European Safety and Reliability Association, ESRA. He is a member of the editorial board of various international sciatic journals on reliability engineering and system safety. He is co-author of four international books, and more than 170 papers in international journals. He serves as referee of several international journals. 
Figure 1: 2D Smolyak points: a) $o q=4$; b) $o q=6$

Figure 2: Transition diagram of the multi-state physics model of crack development in Alloy 82/182 dissimilar metal welds [9]

Figure 3: Time-dependent behavior for $\operatorname{Pi}(t)$, base case [9]

Figure 4: Error between the model output and the PCE approximation, using $d=3$

Figure 5: Time-dependent behavior for $\mathrm{S}_{\mathrm{i}}$, for states 1:initial; 2:microcrack; 3:circumferential; 4:radial; 5:leak;6:rupture

Figure 6: Time-dependent behavior for $\mathrm{S}_{\mathrm{Ti}}$, for states 1:initial; 2:microcrack; 3:circumferential; 4:radial; 5:leak;6:rupture

Figure 7: Time-dependent behavior for $\mathrm{S}_{\mathrm{Ti}}-\mathrm{S}_{\mathrm{i}}$, for states 1:initial; 2:microcrack; 3:circumferential; 4:radial; 5:leak;6:rupture

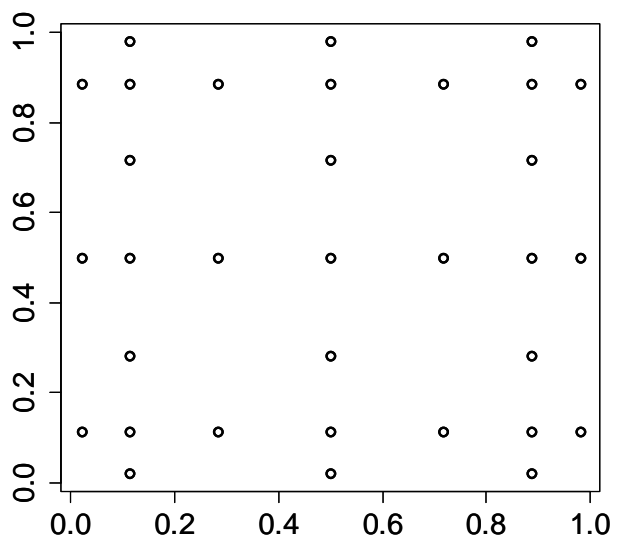

a)

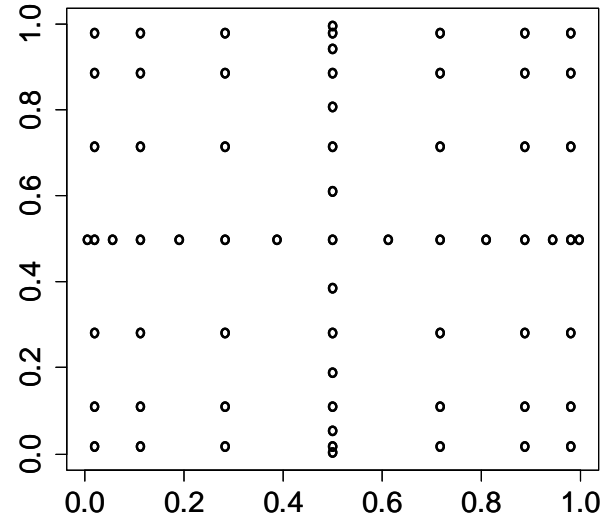

b)

Figure 1: 2D Smolyak points: a) $o q=4$; b) $o q=6$

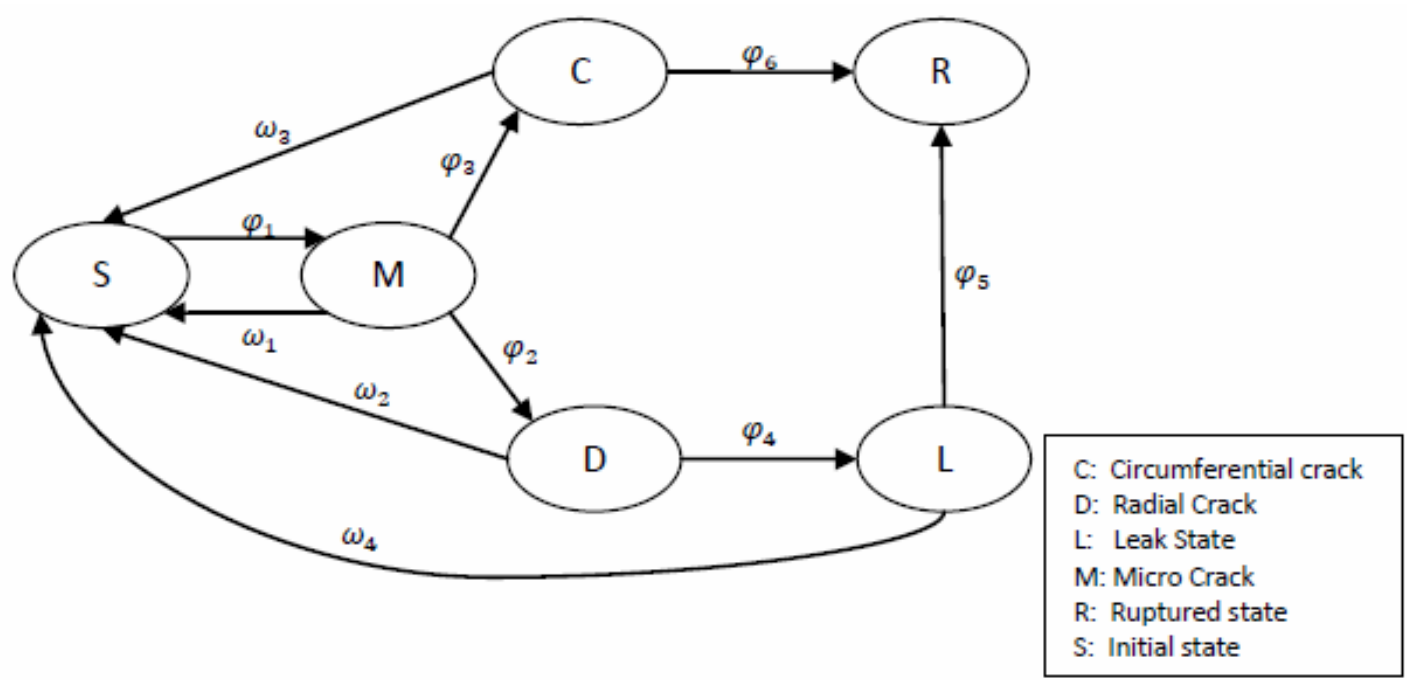

Figure 2: Transition diagram of the multi-state physics model of crack development in Alloy 82/182 dissimilar metal welds [9] 


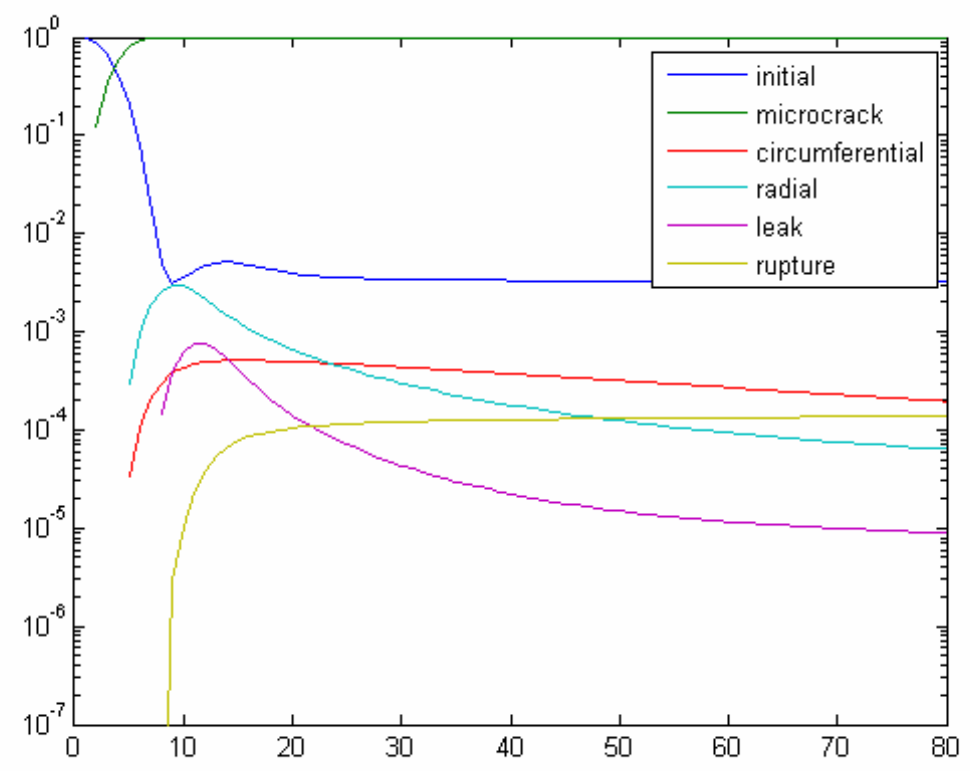

Figure 3: Time-dependent behavior for $\mathrm{P}_{\mathrm{i}}(t)$, base case [9]

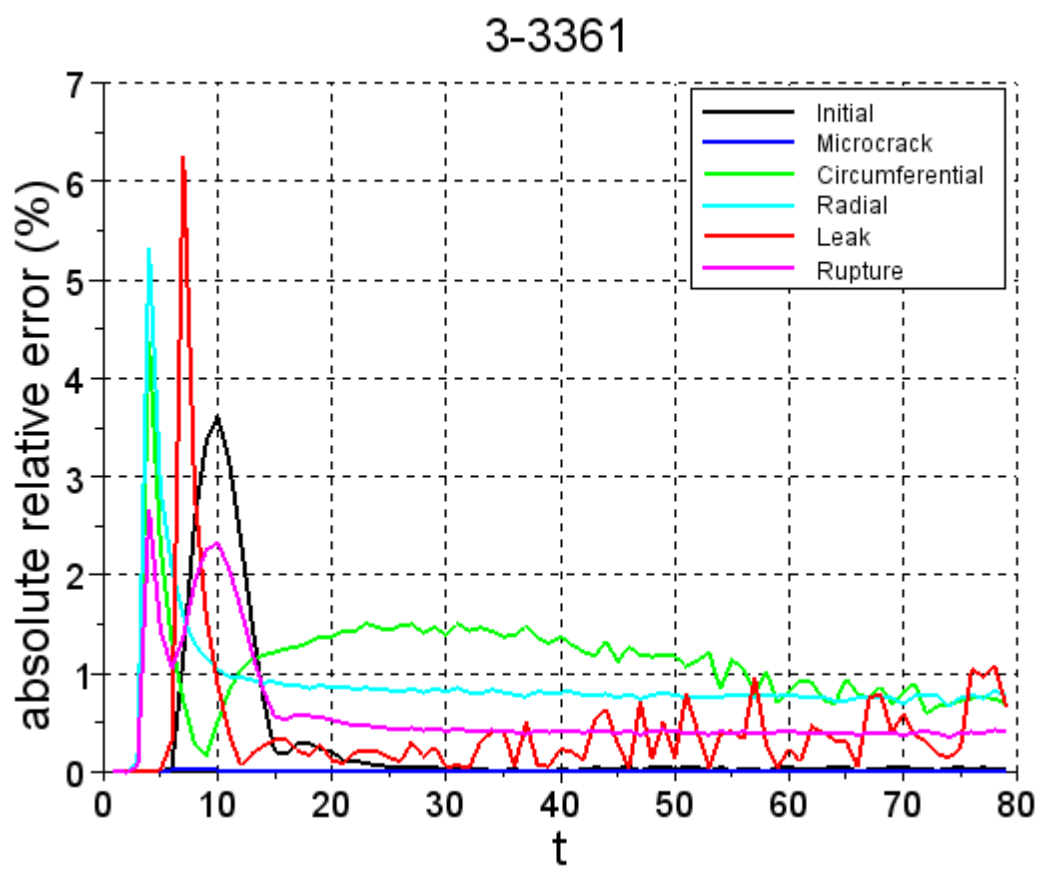

Figure 4: Error between the model output and the PCE approximation, using $d=3$ 


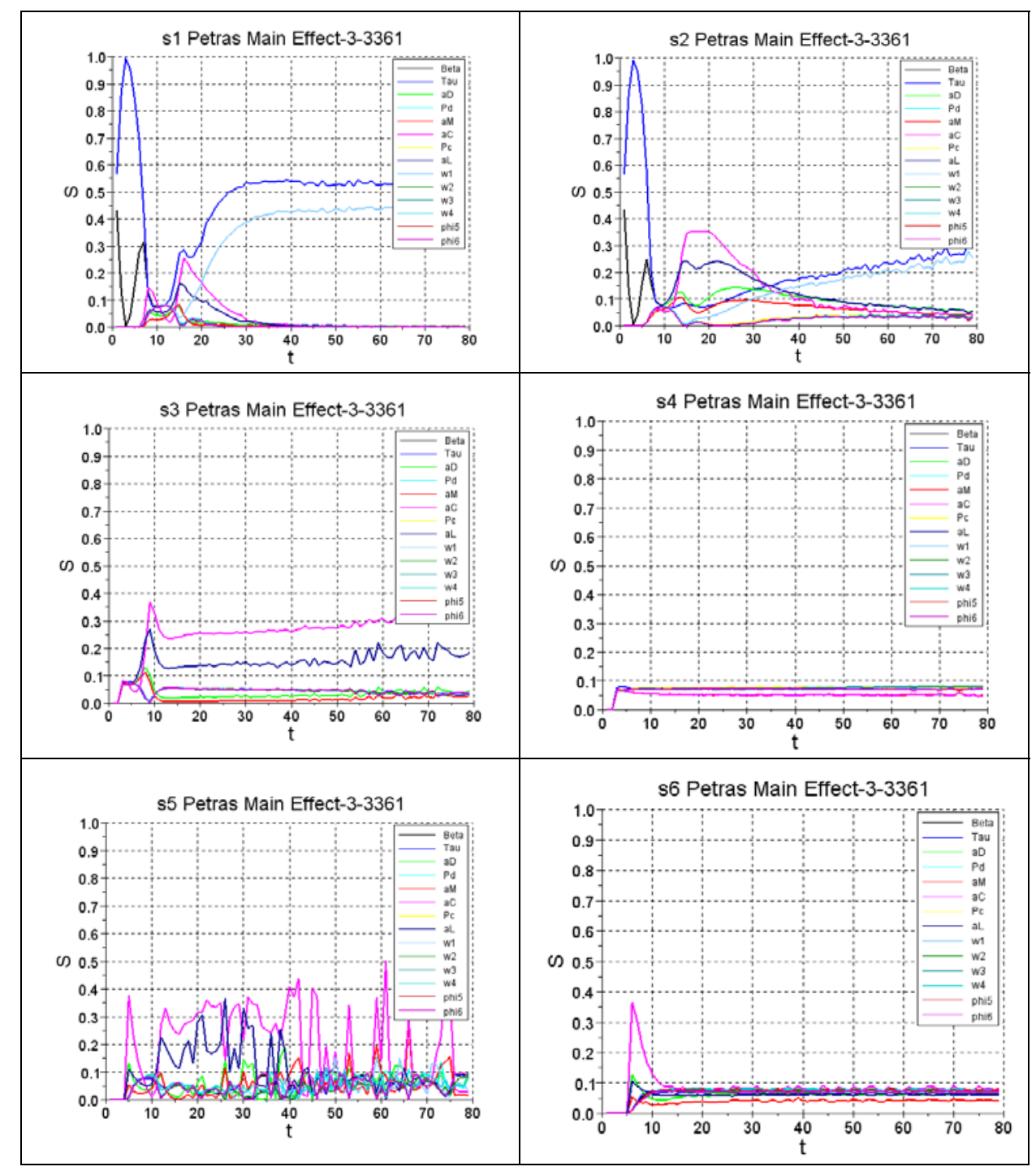

Figure 5: Time-dependent behavior for $\mathrm{S}_{\mathrm{i}}$, for states 1: initial; 2:microcrack; 3:circumferential; 4:radial; 5:leak;6:rupture 


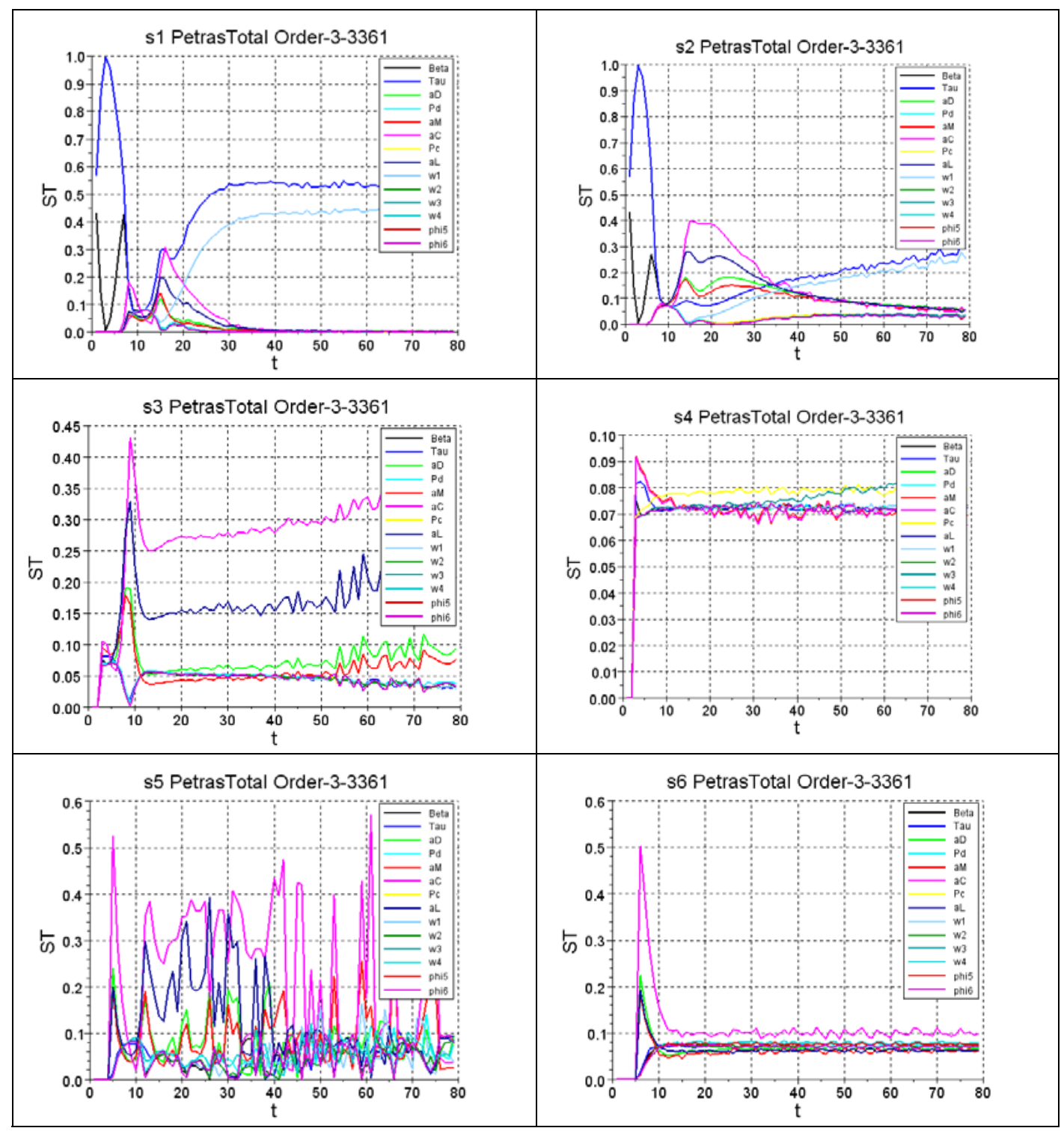

Figure 6: Time-dependent behavior for $\mathrm{S}_{\mathrm{Ti}}$ for states 1: initial; 2:microcrack; 3:circumferential; 4:radial; 5:leak;6:rupture 


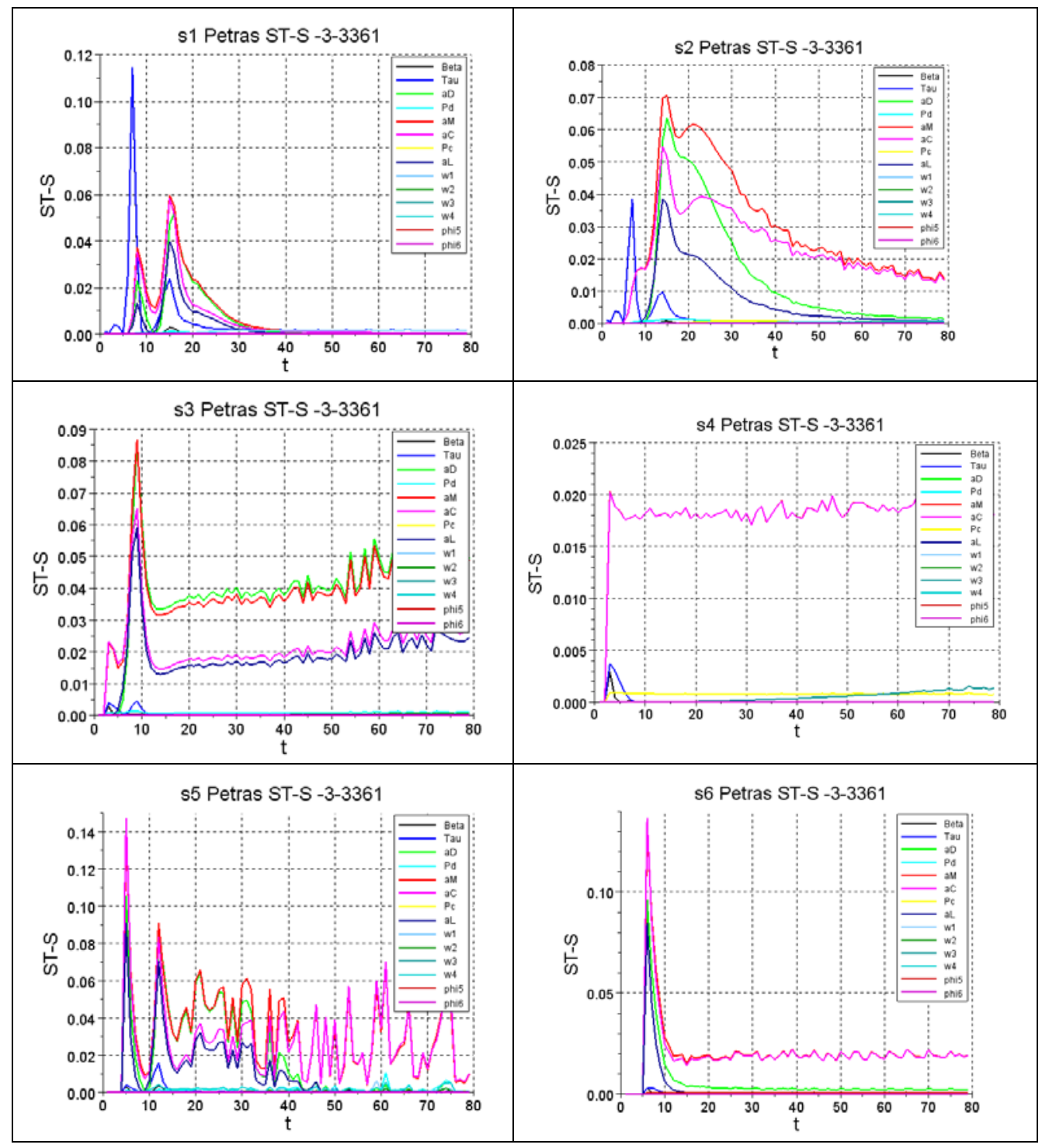

Figure 7: Time-dependent behavior for $\left(\mathrm{S}_{\mathrm{Ti}}-\mathrm{S}_{\mathrm{i}}\right)$ for states 1: initial; 2:microcrack; 3:circumferential; 4:radial; 5:leak;6:rupture 
Table 1: Relation between probability distribution and polynomial basis based on the Wiener-Askey scheme [42]

Table 2: Expressions for transitions rates $\varphi_{1}, \varphi_{2} \varphi_{3}$ and $\varphi_{4}$

Table 3: Parameter definition and base value

Table 4 Important Parameters using a PCE with $d=3$

Table 1: Relation between probability distribution and polynomial basis based on the Wiener-Askey scheme [42]

\begin{tabular}{|c|c|c|}
\hline Distribution & Polynomials & Support \\
\hline Gaussian & Hermite & $\Re$ \\
\hline Uniform & Legendre & {$[-1,1]$} \\
\hline Gamma & Laguerre & {$[0, \infty[$} \\
\hline Beta & Jacobi & {$[-1,1]$} \\
\hline Poisson & Charlier & $\{0,1, \ldots\}$ \\
\hline Negative Binomial & Miexner & $\{0,1, \ldots\}$ \\
\hline Binomial & Krawtchouk & $\{0,1, \ldots N\}$ \\
\hline Hypergeometric & Hahn & $\{0,1, \ldots \mathrm{N}\}$ \\
\hline
\end{tabular}

Table 2: Expressions for transitions rates $\varphi_{1}, \varphi_{2}, \varphi_{3}$ and $\varphi_{4}$

\begin{tabular}{|c|c|}
\hline Transition rate & Expression \\
\hline$\varphi_{1}$ & $(b / \tau)(t / \tau)^{b-1}$ \\
\hline$\varphi_{2}$ & $\begin{array}{cc}\frac{a_{D} P_{D}}{a_{M} u^{2}\left(1-P_{D}\left(1-a_{D} /\left(u a_{M}\right)\right)\right)} & \text { if } u>a_{D} / a_{M} \\
0 & \text { else }\end{array}$ \\
\hline$\varphi_{3}$ & $\begin{array}{cc}\frac{a_{C} P_{C}}{a_{M} u^{2}\left(1-P_{C}\left(1-a_{C} /\left(u a_{M}\right)\right)\right)} & \text { if } u>a_{C} / a_{M} \\
0 & \text { else }\end{array}$ \\
\hline$\varphi_{4}$ & $\begin{array}{cc}\frac{1}{w} & \text { if } w>\left(a_{L}-a_{D}\right) / a_{M} \\
0 & \text { else }\end{array}$ \\
\hline
\end{tabular}


Table 3: Parameter definition and base value

\begin{tabular}{|c|l|c|}
\hline Parameter & \multicolumn{1}{|c|}{ Definition } & Base value \\
\hline$b$ & Weibull shape parameter for crack initiation model & 2.0 \\
\hline$\tau$ & Weibull scale parameter for crack initiation model & 4 years \\
\hline $\mathrm{a}_{\mathrm{D}}$ & Crack length threshold for radial macro-crack & $10 \mathrm{~mm}$ \\
\hline $\mathrm{P}_{\mathrm{D}}$ & Probability that micro-crack evolves as radial crack & 0.009 \\
\hline $\mathrm{a}_{\mathrm{M}}$ & Maximum credible crack growth rate & $9.46 \mathrm{~mm} / \mathrm{yr}$ \\
\hline $\mathrm{a}_{\mathrm{C}}$ & Crack length threshold for circumferential macro-crack & $10 \mathrm{~mm}$ \\
\hline $\mathrm{P}_{\mathrm{C}}$ & $\begin{array}{l}\text { Probability that micro-crack evolves as circumferential } \\
\text { crack }\end{array}$ & 0.001 \\
\hline $\mathrm{a}_{\mathrm{L}}$ & Crack length threshold for leak & $20 \mathrm{~mm}$ \\
\hline$\omega_{1}$ & Repair transition rate from micro-crack & $1 \times 10-3 / \mathrm{yr}$ \\
\hline$\omega_{2}$ & Repair transition rate from radial macro-crack & $2 \times 10-2 / \mathrm{yr}$ \\
\hline$\omega_{3}$ & Repair transition rate from circumferential macro-crack & $2 \times 10-2 / \mathrm{yr}$ \\
\hline$\omega_{4}$ & Repair transition rate from leak & $8 \times 10-1 / \mathrm{yr}$ \\
\hline$\varphi_{5}$ & Leak to rupture transition rate & $2 \times 10-2 / \mathrm{yr}$ \\
\hline$\varphi_{6}$ & Macro-crack to rupture transition rate & $1 \times 10-5 / \mathrm{yr}$ \\
\hline
\end{tabular}

Table 4 Important Parameters using a PCE with $d=3$

\begin{tabular}{|c|c|c|c|c|c|c|}
\hline State & \multicolumn{7}{|c|}{ Parameters } \\
\hline 1 & $b$ & $\tau$ & $\mathrm{a}_{\mathrm{C}}$ & $\omega_{1}$ & $\mathrm{a}_{\mathrm{D}}$ & -- \\
\hline 2 & $\tau$ & $b$ & $\mathrm{a}_{\mathrm{C}}$ & $\mathrm{a}_{\mathrm{L}}$ & $\mathrm{a}_{\mathrm{D}}$ & $\omega_{1}$ \\
\hline 3 & $\mathrm{a}_{\mathrm{C}}$ & $\mathrm{a}_{\mathrm{L}}$ & $\mathrm{a}_{\mathrm{D}}$ & $\mathrm{a}_{\mathrm{M}}$ & -- & -- \\
\hline 4 & -- & -- & -- & -- & -- & -- \\
\hline 5 & $\mathrm{a}_{\mathrm{C}}$ & $\mathrm{a}_{\mathrm{D}}$ & $\mathrm{a}_{\mathrm{M}}$ & $\omega_{1}$ & -- & -- \\
\hline 6 & $\mathrm{a}_{\mathrm{C}}$ & -- & -- & -- & -- & -- \\
\hline
\end{tabular}

